\title{
Current status and recent advances of gene therapy in hematological diseases
}

\author{
Masato Yamamoto ${ }^{1} \cdot$ Kenzaburo Tani $^{2}$
}

Received: 26 May 2016 / Accepted: 27 May 2016 / Published online: 1 June 2016

(C) The Japanese Society of Hematology 2016

Gene therapy and novel therapeutics applying gene therapy-based techniques have experienced a drastic advance in the past 10 years. Clinically functional "gene therapies" are established now for certain diseases, and many other promising therapeutics have been developed, with such a trend being most noticeable in the field of hematological diseases.

Since most hereditary diseases are caused by genetic changes, fixing disease-related mutations has been a straight-forward and logical approach to treat hereditary diseases. However, as reported by Orkin-Motulsky Report in 1995 (http://osp.od.nih.gov/office-biotechnology-activities/orkin-motulsky-report), efficacy of gene delivery has been a large challenge for long span of time. Recent advances in "gene therapy" and "gene therapy-based approaches" have been quite substantial, which include delivery vectors, gene engineering technologies, application to chimeric antigen receptor (CAR) T cell therapy, and oncolytic viruses. These advances have realized many of the unmet promises of gene therapy for genetic diseases. Furthermore, the application of gene therapy technologies to non-hereditary diseases such as malignancies has recently started to display positive outcomes, such as with CAR-T cell therapy and the oncolytic virus.

Interestingly, hematological diseases have been one of the main fields where many novel concepts, approaches, and

Masato Yamamoto

yamam016@umn.edu

1 Division of Basic and Translational Research, Department of Surgery, University of Minnesota, 420 Delaware St. SE. (MMC195), Minneapolis, MN 55455, USA

2 Project Division of ALA Advanced Medical Research, The Institute of Medical Science, The University of Tokyo, Tokyo, Japan technologies are applied first. Many examples can be named easily: cancer stem cell concept, combination chemotherapies, flow cytometry, and gene therapy are among those on the list. The reason behind such a trend may be the easiness of isolation of individual cells exhibiting problems, as well as the relative ease of access of the disease for detection and intervention compared to other diseases in other fields (such as solid tumors and cardiovascular disorders).

For hereditary diseases in hematology, the advance in delivery system (particularly, the advance of the viral vector system) has contributed greatly to the improved clinical outcomes with reduced genotoxicity. Such a trend is most evident in ex vivo gene therapy of hereditary diseases, and the review by Dr. Tani summarizes recent advances in hematological disorders with a special emphasis on global comparisons. Another recent game changer has been the application of gene editing technologies for the cure of human diseases. Zinc finger nucleases, as well as Cas/ CRISPRs and TALENs can be used to edit target genes, achieving knock down or repair. These technologies have great potential to clinically "destroy" or "repair" the mutant gene back to normal without causing genotoxicity in other genes. The basic features of gene editing techniques and their applications to hematological disorders are reviewed by Drs. Osborn, Belanto, Tolar, and Voytas.

On the other hand, the concept of "gene therapy" has been expanded to acquired diseases such as malignancies. The correction of $100 \%$ of malignant cells is difficult to achieve in actively proliferating cells and the normalization of the initial inducer mutation does not necessarily kill all malignant cells, and thus treatments are preferred to be designed for elimination of malignant cells by the therapeutic modalities based on gene therapy delivery technologies, not by simple mutation repair. Recent advance of CAR-T cell therapy, including its application to B cell 
malignancies, is a lead example of such an approach. The development and application of CAR-T cells are reviewed by Drs. Davila and Sadelain. Gene delivery methodology has also been applied to malignancies from a variety of angles. Immuno-gene therapy (such as GVAX, GM-CSFproducing whole tumor cell vaccine) has shown promising results, RNA interference has been actively tested, and suicide gene-based regulation of GVHD has been tested for hematological malignancies. In addition, oncolytic virus is acquiring greater attention after oncolytic herpesvirus (T-VEC) was approved as a drug in 2015. These efforts are reviewed by Dr. Domingo-Musibay and Dr. Yamamoto.

As discussed in each review, gene therapy and gene therapy-based therapeutic approaches have made significant advances in recent years. Hematology is one of the fields where rapid advance and broader clinical applications are expected, and some of the therapies are becoming relatively standard treatments already. In addition, many newly developed, promising therapies are waiting for clinical application. We expect that the advance of therapeutic approaches based on gene therapy in the coming years will be even faster than the past 10 years. We hope these reviews provide quick insights to recent developments of gene therapy and new therapeutics arriving to clinics in the near future.

The guest editors thank all review authors for contribution, the Chief Editor, Dr. Kitamura, for this opportunity, and the Journal Office of International Journal of Hematology for editorial work and patience. 